\title{
Start Low and Go Slow
}

Tiffany Chan, MD; Lana Shaker, MD; Lewis S. Nelson, MD

\section{A woman in her third decade with no known \\ medical history presented to the ED \\ for evaluation of depressed mental status.}

Case

A woman in her third decade with no known medical history was dropped off at

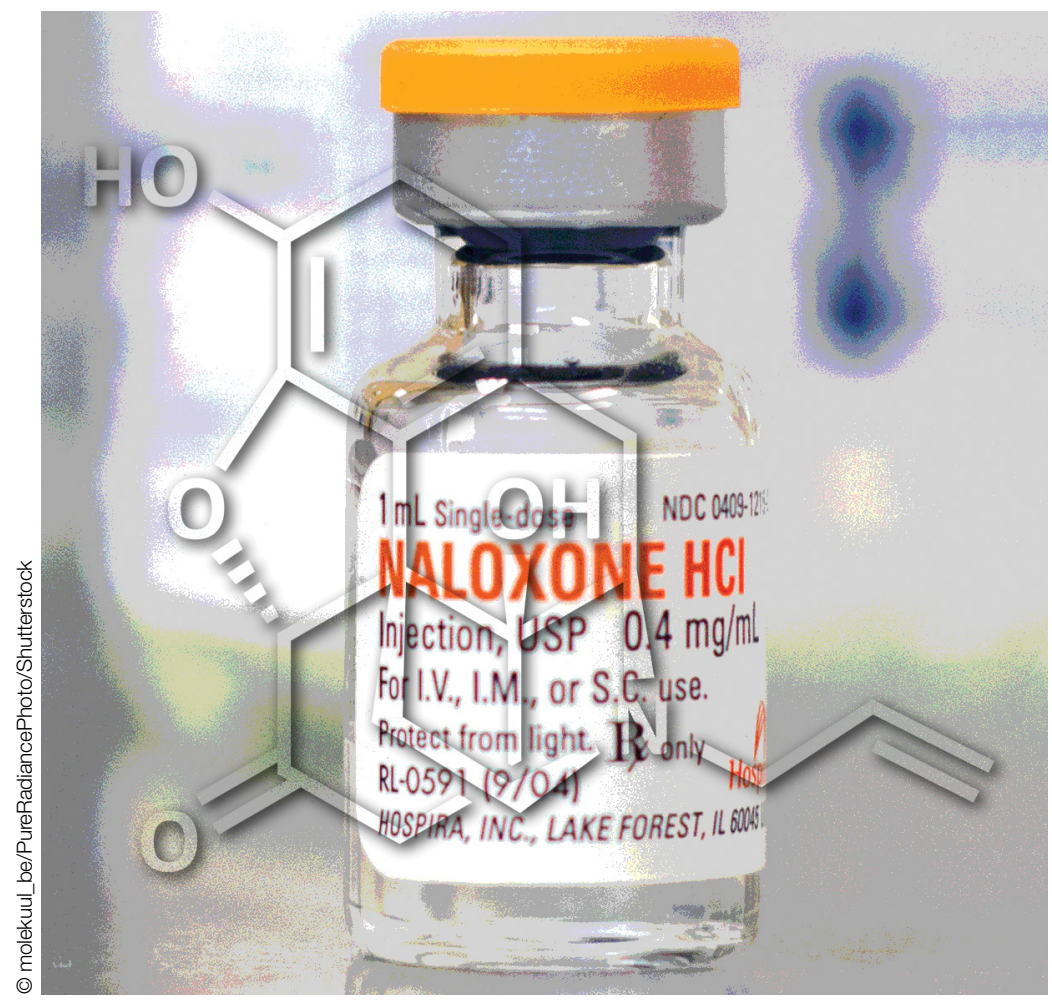

the waiting area of the ED for evaluation of depressed mental status. Upon arrival, the patient was unresponsive and cyanotic, with a pulse oximetry of $65 \%$ on room air. Bag-valve mask (BVM) ventilation rapidly improved oxygen saturation to $90 \%$. The patient's other vital signs were: heart rate, 141 beats/min; blood pressure (BP), 117/65 $\mathrm{mm} \mathrm{Hg}$; and temperature, afebrile.

Upon examination, the patient's pupils were pinpoint and her ventilatory effort was shallow, leading the emergency physician (EP) to suspect the patient's depressed mental status was due to an opioid overdose.

The patient was given $2 \mathrm{mg}$ of intravenous (IV) naloxone, after which she became more alert and responsive, with improved respiratory effort. After receiving naloxone, the patient vomited copiously. Pulmonary examination revealed diffuse rales, most prominently at the right lung base, and a cough productive of thick sputum.

During the patient's course in the ED, she became increasingly hypotensive with systolic BP readings around $70 \mathrm{~mm}$ $\mathrm{Hg}$; tachycardia, fluctuating at around 120 beats/min; and persistent hypoxia of

Dr Chan is a third-year resident, department of emergency medicine, Rutgers New Jersey Medical School, Newark. Dr Shaker is a first-year resident, department of emergency medicine, Rutgers New Jersey Medical School, Newark. Dr Nelson, editor of "Case Studies in Toxicology," is professor and chair of the department of emergency medicine, Rutgers New Jersey Medical School; and a medical toxicologist, New Jersey Poison Information \& Education System, both in Newark. He is also associate editor, toxicology, of the EMERGENCY MEDICINE editorial board.

Authors' Disclosure Statement: The authors report no actual or potential conflict of interest in relation to this article.

DOI: 10.12788/emed.2017.0072 
$90 \%$ saturation on a nonrebreather mask. A chest X-ray demonstrated pulmonary edema with a continuous diaphragm sign suggesting pneumomediastinum. A computed tomography (CT) scan of the chest confirmed pulmonary edema with extensive pneumomediastinum, and the patient was admitted to the intensive care unit (ICU).

\section{What is naloxone and why is it used?}

Naloxone is a nonselective, short-acting, pure opioid antagonist that works at the mu, kappa, and sigma receptors, with the highest affinity for the mu receptor. It is a competitive opioid receptor antagonist that has an elimination half-life of approximately 30 minutes. Though naloxone was originally developed to reverse the effects of anesthesia postoperatively, ${ }^{1}$ today it is more commonly used to treat ventilatory depression in patients whose clinical findings are most likely due to an opioid overdose.

What is acute opioid withdrawal syndrome? Opioid-dependent individuals who abstain from use for more than a few hours generally develop opioid withdrawal syndrome (OWS). The effects of OWS include mild-to-moderate tachycardia and hypertension, nausea, vomiting, piloerection, rhinorrhea, and agitated behavior. However, when opioid-dependent patients receive naloxone, OWS develops at a much faster rate (ie, seconds after naloxone administration) and is often more severe.

Findings of naloxone-precipitated OWS include pronounced vital sign abnormalities, seizures, pulmonary edema, and cardiac arrhythmias such as ventricular tachycardia. ${ }^{2}$ These latter findings are primarily due to the sudden release of catecholamines. ${ }^{3}$ In addition, patients suffer the psychological pangs of withdrawal, including dysphoria and drug craving, which often leads to poor decision-making as they search for additional opioids to alleviate these troubling effects.

\section{What determines response to naloxone and development of OWS?}

The severity of precipitated OWS following naloxone administration is determined by both the degree of the patient's opioid dependency and the dosage and rate at which naloxone is given. The depth of opioid dependence is determined to a large extent by the quantity of opioid regularly used and the frequency of exposure. For example, a patient who takes $30 \mathrm{mg}$ of oxycodone daily will likely demonstrate mild OWS, while one who uses $300 \mathrm{mg}$ daily will demonstrate more severe OWSwhether due to abstinence or naloxone.

In addition, longer exposure time of the patient's brain to opioids increases the dependency level. Continuous use of extended-release opioids or methadone, which are both of long duration, essentially "bathe" the brain receptors in opioid around the clock, whereas short-acting opioids, such as fentanyl or heroin, cause peaks and troughs in brain concentrations throughout the day. These trough periods reduce dependency, but increase the abuse liability of the opioid. Patients who only use opioids on the weekend, for example, will have minimal or no OWS following naloxone administration, nor will the toddler with an exploratory ingestion of an opioid medication found in the home. It is therefore important to gauge the extent of a patient's opioid use to improve the safe use of naloxone in the ED.

\section{What is the optimal dosing of naloxone and proper patient management?}

It is essential for clinicians to remember that the ultimate goal of naloxone administration in the ED is to reverse ventilatory depression-not to restore a patient to a normal mental status. ${ }^{4}$ In fact, full awakening, in addition to precipitating OWS, may lead to difficult interpersonal situations in the ED, since such patients often insist on leaving the ED before the effects of naloxone wear off. This situation places the EP in the undesirable position of discharging 
a patient who may predictably relapsethough unlikely to die-after release. ${ }^{5}$

Management in the Hospital Setting. Given the advanced medical care environment in a hospital, the approach to opioid overdose patients can be metered. This means providing temporary noninvasive mechanical ventilatory support through BVM or laryngeal mask airways, which allow both oxygenation and ventilation (reducing the patient's partial pressure of carbon dioxide), prior to giving naloxone. ${ }^{6}$ Studies on animal models have shown that lowering the partial pressure of carbon dioxide reduces the catecholamine response to naloxone. ${ }^{7}$

Although recent literature and textbook recommendations regarding naloxone dosages vary, ${ }^{1}$ the safest initial dose of naloxone in the hospital setting is $0.04 \mathrm{mg}$ (40 $\mathrm{mcg}$ ) IV, or $0.08 \mathrm{mg}(80 \mathrm{mcg})$ intramuscularly (IM). ${ }^{8}$ Whether given by IV or IM route, frequent reassessment of the adequacy of spontaneous ventilatory effort and oxygenation are required.

While the rate of opioid reversal is slower when giving lower doses of naloxone, this approach reduces the severity of precipitated OWS. In fact, in most patients who receive low-dose naloxone administration will not awaken but will develop life-sustaining spontaneous ventilation. ${ }^{8}$

By monitoring of the patient's ventilatory rate and depth, along with capnometry and pulse oximetry (without providing exogenous oxygen), the EP can identify the need for additional naloxone. Since the half-life of naloxone is shorter than that of many opioids, proper ventilatory monitoring is essential to assess for the waning of naloxone's effects and return of respiratory depression.

Treatment in the Nonhospital Setting. Emergency medical service (EMS) workers typically, and often by situational necessity, approach opioid overdose patients more aggressively than do EPs in the ED. Although some EMS systems utilize the IV route, most EMS workers, like laypersons, administer an initial naloxone dose of 0.4 mg IM or 2 or $4 \mathrm{mg}$ intranasally (IN). Due to the slower rate of absorption and lower bioavailability (with IN administration), both IM and IN naloxone equate to roughly $0.08 \mathrm{mg}$ IV.

For patients in whom there is no risk for opioid dependence, the initial dose of naloxone is relatively inconsequential, and higher doses can be safely administered. However, for most patients, including those in the ED setting, in whom one cannot be certain of their depth of dependence, the safest approach is to "start low and go slow" with naloxone administration, while providing supportive care.

\section{Case Conclusion}

The patient was not opioid-naïve, explaining the catecholamine surge and related cardiovascular dysfunction and pulmonary edema. The pneumomediastinum and pulmonary aspiration were due to the violent retching and vomiting. After being admitted to the ICU, the patient was started on vancomycin and piperacillin/tazobactam for empiric coverage for mediastinal emphysema. She was kept NPO, assessed by cardiothoracic surgery, and treated with gentle fluid hydration.

A repeat CT showed a stable pneumomediastinum. Her hypoxia, tachycardia, and hypotension gradually improved over about 6 hours. The following day, the patient's mental status normalized, and she discharged herself from the hospital against medical advice.

\section{References}

1. Connors NJ, Nelson LS. The evolution of recommended naloxone dosing for opioid overdose by medical specialty. J Med Toxicol. 2016;12(3):276-281. doi:10.1007/s13181-016-0559-3.

2. Lameijer, H, Azizi N, Ligtenberg JJ, Ter Maaten JC. Ventricular tachycardia after naloxone administration: a drug related complication? Case report and literature review. Drug Saf Case Rep. 2014;1(1):2. doi:10.1007/s40800-014-0002-0.

3. Kienbaum P, Thürauf N, Michel MC, Scherbaum N, Gastpar M, Peters J. Profound increase in epinephrine concentration in plasma and cardiovascular stimulation after mu-opioid receptor blockade in opioid-addicted patients during barbiturate-induced anesthesia for acute detoxification. Anesthesiology. 1998;88(5):1154-1161.

4. Kim HK, Nelson LS. Reducing the harm of opioid 


\section{Continued from page 556}

overdose with the safe use of naloxone: a pharmacologic review. Expert Opin Drug Saf. 2015;14 (7):11371146. doi:10.1517/14740338.2015.1037274.

5. Willman MW, Liss DB, Schwarz ES, Mullins ME. Do heroin overdose patients require observation after receiving naloxone? Clin Toxicol (Phila). 2017;55(2):8187. doi:10.1080/15563650.2016.1253846.

6. Boyer EW. Management of opioid analgesic overdose. N Engl J Med. 2012;367(2):146-155.
doi:10.1056/NEJMra1202561.

7. Mills CA, Flacke JW, Miller JD, Davis LJ, Bloor BC, Flacke WE. Cardiovascular effects of fentanyl reversal by naloxone at varying arterial carbon dioxide tensions in dogs. Anesth Analg. 1988;67(8):730-736.

8. Kim HK, Nelson LS. Reversal of opioid-induced ventilatory depression using low-dose naloxone $(0.04$ mg): a case series. J Med Toxicol. 2015;12(1):107-110. doi:10.1007/s13181-015-0499-3. 